\title{
Cosmology of surviving Horndeski theory: The road ahead
}

\author{
Noemi Frusciante, ${ }^{1}$ Simone Peirone, ${ }^{2}$ Santiago Casas, ${ }^{3}$ and Nelson A. Lima ${ }^{4}$ \\ ${ }^{1}$ Instituto de Astrofísica e Ciências do Espaço, Faculdade de Ciências da Universidade de Lisboa, \\ Edificio C8, Campo Grande, P-1749016 Lisboa, Portugal \\ ${ }^{2}$ Institute Lorentz, Leiden University, PO Box 9506, Leiden 2300 RA, The Netherlands \\ ${ }^{3}$ AIM, CEA, CNRS, Université Paris-Saclay, Université Paris Diderot; \\ Sorbonne Paris Citè, F-91191 Gif-sur-Yvette, France \\ ${ }^{4}$ ITP, Ruprecht-Karls-Universitt Heidelberg, Philosophenweg 16, 69120 Heidelberg, Germany
}

(Received 6 November 2018; published 29 March 2019)

\begin{abstract}
In the context of the effective field theory of dark energy (EFT) we perform agnostic explorations of Horndeski gravity. We choose two parametrizations for the free EFT functions, namely, a power law and a dark energy density-like behavior on a nontrivial Chevallier-Polarski-Linder background. We restrict our analysis to those EFT functions which do not modify the speed of propagation of gravitational waves. Among those, we prove that one specific function cannot be constrained by data since its contribution to the observables is below the cosmic variance, although we show it has a relevant role in defining the viable parameter space. We place constraints on the parameters of these models by combining measurements from present-day cosmological data sets, and we prove that the next-generation galaxy surveys can improve such constraints by 1 order of magnitude. We then prove the validity of the quasistatic limit within the sound horizon of the dark field, by looking at the phenomenological functions $\mu$ and $\Sigma$, associated, respectively, with clustering and lensing potentials. Furthermore, we notice up to $5 \%$ deviations in $\mu, \Sigma$ with respect to general relativity at scales smaller than the Compton one. For the chosen parametrizations and in the quasistatic limit, future constraints on $\mu$ and $\Sigma$ can reach the $1 \%$ level and will allow us to discriminate between certain models at more than $3 \sigma$, provided the present best-fit values remain.
\end{abstract}

DOI: $10.1103 /$ PhysRevD.99.063538

\section{INTRODUCTION}

The attempt to find a definite theory of gravity able to explain the late-time acceleration of the Universe has resulted in a wide selection of dark energy (DE) and modified gravity (MG) models [1-6]. When exploring the cosmology of these models, it is very useful to employ a unified approach to describe in a model-independent fashion any departure from general relativity (GR). Among the many approaches presented in the literature, a popular framework is the one based on the $\mu, \Sigma$ parametrization $[7,8]$, according to which deviations from GR in the Poisson and lensing equations are encoded, respectively, in the $\mu$ and $\Sigma$ phenomenological functions. However, one has to rely on the quasistatic (QS) approximation in order to express these functions in an analytical form for a chosen theory. For this reason, the approach has a limitation given by the breakdown scale of the QS assumption. Such a scale, usually identified with the cosmological horizon, has been claimed to be instead the sound horizon of the dark field [9].

Another general framework, encompassing theories with one additional scalar degree of freedom (DOF), is the effective field theory of dark energy (EFT) [10,11]. Such a description parametrizes the evolution of linear cosmological perturbations in terms of a few free functions of time, dubbed EFT functions. The benefit of using the EFT approach relies on a direct connection with the underlying theory of gravity. Indeed, each EFT function multiplies a specific geometrical operator in the action: Thus, picking out a set of EFT functions translates into selecting a class of DE/MG models. Moreover, the mapping procedure, which allows us to translate a specific theory in the EFT language, does not rely on any QS approximation [10-16]. An resembling basis of the EFT functions is the $\alpha$-basis [16-18]. In the latter, the free functions can be directly related to some phenomenological aspects of the DE field, such as the running of the Planck mass, braiding and kineticity effects, and deviation in the speed of propagation of tensor modes [17].

In the present work, we perform a cosmological investigation by means of agnostic parametrizations in terms of the EFT functions. We select the subset of EFT functions describing the Horndeski theory [19] (or generalized Galileon [20]). In particular, we consider the class of models satisfying the condition $c_{t}^{2}=1$, which accommodates the stringent bound on the speed of propagation of tensor modes placed by the LIGO and VIRGO collaborations after the detection of the gravitational wave (GW) event GW170817 and its optical counterpart [21-23]. 
The implication of this result on modified gravity theories has been discussed in several works [24-34] and, in the case of Horndeski theory, the surviving viable action involves a reduced number of free functions [24]. In particular, the quintic Lagrangian is removed, and the coupling with the Ricci scalar in the quartic Lagrangian reduces to a general function of the scalar field. Hereafter, we refer to such an action as the "surviving" Horndeski action ( $\mathrm{sH})$. Very recently it has been shown that it is possible to build a class of theories where the GW speed is set to unity dynamically when the scalar is decoupled from the matter sector [35]. However, it is worth noticing that the applicability of the GW constraint is still the subject of debate since, as pointed out in Ref. [36], the energy scales detected by LIGO lie very close to the typical cutoff of many DE models.

In the next decade, several large-scale surveys, such as DESI, Euclid, SKA and LSST, are planned to start, and they will cover the entire redshift range over which dark energy played a significant role in the accelerated expansion. Looking forward to having real data, forecasts analysis is improving our knowledge of cosmology by looking at specific gravity models as well as modelindependent parametrizations [37-41]. In this work we provide cosmological constraints on $\mathrm{sH}$ theories using both present data sets and future spectroscopic galaxy clustering (GC) and weak lensing (WL) observables. We show how the latter are able to set tighter constraints on the parameters entering the $\mathrm{sH}$ action.

The paper is organized as follows. In Sec. II, we give an overview of the $\mathrm{sH}$ theory and its parametrizations in the EFT formalism. In Sec. III, we introduce the agnostic parametrizations defining the $\mathrm{sH}$ models, the codes and data sets used for the Monte Carlo Markov chain analysis, as well as WL and GC forecasts. In Sec. IV, we discuss the results and present the constraints on the model parameters from present and future surveys. Finally, we conclude in Sec. V.

\section{THEORY}

\section{A. Horndeski theory and its parametrizations}

Horndeski theory has become very popular, as it is the most general scalar tensor theory in four dimensions constructed from the metric $g_{\mu \nu}$, the scalar field $\phi$ and their derivatives, giving second order field equations. Its generality relies on a certain number of free functions in the action, namely, $\left\{\mathcal{K}, G_{3}, G_{4}, G_{5}\right\}[\phi, X]$, where $X=\partial^{\mu} \phi \partial_{\mu} \phi$. The number of these functions was reduced after the detection of the GW170817 event. Indeed, the stringent constraint on the speed of propagation of the tensor modes disfavors the presence of the $G_{5}$ term and reduces $G_{4}$ to solely a function of the scalar field [24]. Thus, the sH action, which assumes an unmodified speed of propagation of gravitational waves $\left(c_{t}^{2}=1\right)$, takes the following form:
$\mathcal{S}_{s H}=\int d^{4} x \sqrt{-g}\left[\mathcal{K}(\phi, X)+G_{3}(\phi, X) \square \phi+G_{4}(\phi) R\right]$,

where $g$ is the determinant of the metric $g_{\mu \nu}$ and $R$ is the Ricci scalar. Even though the Horndeski action drastically simplifies, a high degree of freedom in choosing the above functions still remains.

We are interested in investigating the linear cosmological perturbations; thus, in the following we focus on a complementary framework to describe the sH action, i.e., the EFT approach [10,11]. Within this framework we can write the corresponding linear perturbed action around a flat Friedmann-Lemaitre-Robertson-Walker (FLRW) background and in unitary gauge, which reads

$$
\begin{aligned}
\mathcal{S}= & \int d^{4} x \sqrt{-g}\left\{\frac{m_{0}^{2}}{2}[1+\Omega(a)] R+\Lambda(a)-c(a) a^{2} \delta g^{00}\right. \\
& \left.+m_{0}^{2} H_{0}^{2} \frac{\gamma_{1}(a)}{2}\left(a^{2} \delta g^{00}\right)^{2}-m_{0}^{2} H_{0} \frac{\gamma_{2}(a)}{2} a^{2} \delta g^{00} \delta K\right\},
\end{aligned}
$$

where $m_{0}^{2}$ is the Planck mass, $\delta g^{00}$ and $\delta K$ are the perturbations, respectively, of the upper time-time component of the metric and the trace of the extrinsic curvature, $H_{0}$ is the Hubble parameter at present time, and $a$ is the scale factor. Here, $\left\{\Omega, c, \Lambda, \gamma_{1}, \gamma_{2}\right\}$ are the so-called EFT functions. Note that $\Lambda$ and $c$ can be expressed in terms of $\Omega$, the conformal Hubble function $\mathcal{H}$, and the densities and pressures of matter fluids by using the background field equations $[10,11]$. Thus, we are left with only three free EFT functions. While $\Omega$ acts at both background and perturbation levels, $\gamma_{1}$ and $\gamma_{2}$ contribute only to the linear perturbation evolution.

The EFT functions can be specified for a chosen theory once the mapping has been worked out [10-16]. For action (1), the mapping simply reads

$$
\begin{aligned}
1+\Omega= & \frac{2}{m_{0}^{2}} G_{4}, \\
m_{0}^{2} H_{0}^{2} \gamma_{1}= & \mathcal{K}_{X X} X^{2}-3 \frac{\mathcal{H}}{a^{6}} G_{3 X X} \dot{\phi}^{5}-G_{3 \phi X} \frac{\dot{\phi}^{4}}{2 a^{4}} \\
& +G_{3 X} \frac{\dot{\phi}^{2}}{2 a^{4}}(\ddot{\phi}+2 \mathcal{H} \dot{\phi}), \\
m_{0}^{2} H_{0} \gamma_{2}= & -2 G_{3 X} \frac{\dot{\phi}^{3}}{a^{3}},
\end{aligned}
$$

where dots are derivatives with respect to conformal time, $\tau$, and the subscripts $X$ and $\phi$ are, respectively, the derivatives with respect to $X$ and $\phi$. Therefore, the EFT approach practically translates the problem of choosing appropriate forms for the $\mathcal{K}, G_{i}$-functions into choosing specific forms of the EFT functions. 
Let us now comment on the functional dependence of the $\mathcal{K}, G_{i}$-functions. All of them can modify the expansion history regardless of their specific dependence on $\phi$ or $X$. However, this is not true at the level of perturbations. The following are some examples:

(i) $G_{3}$-function. $G_{3}=G_{3}(\phi)$ : This function solely affects the expansion history in the form of a dynamical DE. Indeed, it can be recast as an equivalent contribution of $\mathcal{K}$ in the form $\mathcal{K}=$ $F(\phi) X$ by integration by parts (with $F \propto G_{3 \phi}$ ) [42]. $G_{3}=G_{3}(\phi, X)$ : This function gives nonvanishing $\gamma_{1}$ and $\gamma_{2}$. Note that if $\gamma_{2} \neq 0$, the function $\gamma_{1}$ is forced to be nonzero from Eq. (3) (except in the case of fine-tuning). The opposite does not hold. This is an important aspect when selecting the combinations of nonvanishing EFT functions. Finally, $G_{3 X} \neq 0$ has been identified as responsible for the braiding effect or mixing of the kinetic terms of the scalar and metric [42]. For this reason, $\gamma_{2}$ can be interpreted as a braiding function. Thus, in order to parametrize for, e.g., the so-called kinetic gravity braiding models (KGB) [42], both $\gamma_{1}$ and $\gamma_{2}$ need to be active.

(ii) $G_{4}$-function. When $G_{4} \neq m_{0}^{2} / 2$, it is the only function which can modify the coupling, i.e., $\Omega \neq 0$. The function $m_{0}^{2}(1+\Omega)$ can be interpreted as an effective Planck mass, and its evolution rate can be defined as $\alpha_{M}=\dot{\Omega} / \mathcal{H}(1+\Omega)$ [17]. A running Planck mass also contributes to the braiding effect: In particular, in the case $G_{3 X}=0$, the running Planck mass is solely responsible for the braiding effect [17].

(iii) $\mathcal{K}$-function. When $\mathcal{K}$ is only a function of $\phi$, it does not give any contribution to the perturbations: In fact, $\gamma_{1}$ does not depend on $\mathcal{K}(\phi)$. On the contrary, when $\mathcal{K}=\mathcal{K}(\phi, X)$, it contributes both to the background equations and to the perturbations through $\gamma_{1}$ (the latter if $\mathcal{K}_{X X} \neq 0$ ). In particular, in the case $\left\{G_{4}(\phi), G_{3}=0, \mathcal{K}(X)\right\}$ and $\mathcal{K}_{X X} \neq 0$, the form of $\gamma_{1}$ is fixed in terms of background functions as $\gamma_{1}=\frac{c}{m_{0}^{2} H_{0}^{2}}\left(\frac{\dot{c}}{\dot{\Lambda}}-1\right)$.

In the regime in which the QS approximation holds, it has been found that $\gamma_{1}$ is negligible for linear cosmological perturbations [11,12]. In Sec. III A, we show that although $\gamma_{1}$ is unlikely to be constrained by cosmological data, it still plays a relevant role in defining the stable parameter space of the theory.

In order to study the cosmological signatures of each EFT function, we introduce the $\mu, \Sigma$ parametrization, which allows us to encode all possible deviations from GR at the level of the linear perturbed field equations $[7,8]$. They are defined, respectively, as the deviations from the GR Poisson equation and the GR lensing equation and, in Fourier space, they read

$$
\begin{aligned}
-k^{2} \psi & =4 \pi G_{N} a^{2} \mu(a, k) \rho \Delta, \\
-k^{2}(\psi+\phi) & =8 \pi G_{N} a^{2} \Sigma(a, k) \rho \Delta,
\end{aligned}
$$

where $\left\{\psi\left(t, x^{i}\right), \phi\left(t, x^{i}\right)\right\}$ are the gravitational potentials, $G_{N}$ is the Newtonian gravitational constant, and $\rho \Delta=$ $\sum_{i} \rho_{i} \Delta_{i}$ includes the contributions of all fluid components. GR is recovered for $\mu=\Sigma=1$.

Although their definition is very general, their explicit and analytical expressions can be found by considering a specific Lagrangian describing a chosen gravity theory with one extra scalar DOF, in the QS approximation $[17,43]$. In such an approximation and for the case under analysis, they read

$$
\begin{aligned}
\mu(a, k) & =\frac{1}{1+\Omega} \frac{1+M^{2} \frac{a^{2}}{k^{2}}}{g_{1}+M^{2} \frac{a^{2}}{k^{2}}}, \\
\Sigma(a, k) & =\frac{1}{2(1+\Omega)} \frac{1+g_{2}+2 M^{2} \frac{a^{2}}{k^{2}}}{g_{1}+M^{2} \frac{a^{2}}{k^{2}}},
\end{aligned}
$$

where $g_{i}$ and $M$ are functions of $a$ and can be expressed in terms of EFT functions, i.e., $\Omega$ and $\gamma_{2}$. As anticipated before, $\gamma_{1}$ does not enter into these expressions because they have been derived in the QS approximation (see Ref. [43] for their explicit expressions and a general discussion; here we address the specific case $c_{t}^{2}=1$ ). Note that $M$ represents the mass of the dark field and, from Eq. (5), we see that it is responsible for the scale dependence of the phenomenological functions: It defines a new scale associated with the extra DOF, i.e., the Compton scale $\left(\lambda_{C} \sim 1 / M\right)$. In the super-Compton limit, i.e., $k / a \ll M$ (subscript “0”), one gets $\mu_{0}=1 /(1+\Omega$ ), $\Sigma_{0}=\mu_{0}$. In this limit, the only signature of modification to gravity comes from the coupling function $\Omega$. Such a function impacts the clustering and lensing potentials and has effects on the cosmic microwave background (CMB) lensing and galaxy weak lensing. Additionally, because of the late time integrated Sachs-Wolfe (ISW) effect, it affects the amplitude of the low-multipole CMB anisotropies. Finally, because of stability conditions (i.e., avoidance of ghost instability for tensor modes [16]), we have $1+\Omega>0$; thus, both $\mu_{0}$ and $\Sigma_{0}$ are positive. In the subCompton limit (subscript " $\infty$ ") both the expressions involve $\gamma_{2}$ and $\Omega$. As in the previous case, $\mu_{\infty}$ and $\Sigma_{\infty}$ are modified, and if $\gamma_{2} \neq 0$ it follows that $\mu_{\infty} \neq \Sigma_{\infty}$. In this case, the effects on the observables are the same as in the previous limit, but they are the results of the combination of both $\Omega$ and $\gamma_{2}$. At these scales, the gravitational slip parameter, $\eta=2 \Sigma / \mu-1$, is modified only if $\gamma_{2} \neq 0$, allowing for the presence of an anisotropic stress term related to the viscosity of a DE fluid [44]. On the other hand, if $\gamma_{2}=0$ it follows that $\mu_{\infty}=\Sigma_{\infty}$ and $\eta_{\infty}=1$. For stability requirements [17], $\mu_{\infty}$ is positive, while a 
conclusion about $\Sigma_{\infty}$ is not straightforward. In this regard, it has been shown in [45] that $(\mu-1)(\Sigma-1) \geq 0$.

In Sec. IV, we verify the applicability of the QS approximation within the sound horizon of the dark field for the specific models analyzed in this work.

\section{METHOD}

\section{A. Models}

In this section, we present two agnostic parametrizations of the EFT functions along with that of the equation of state parameter, $w_{\mathrm{DE}}$, which fixes the expansion history. Then, the underlying theory is fully specified [46].

We employ the DE equation of state given by the Chevallier-Polarski-Linder (CPL) parametrization $[47,48]$ :

$$
w_{\mathrm{DE}}(a)=w_{0}+w_{a}(1-a),
$$

where $w_{0}$ and $w_{a}$ are constants and indicate, respectively, the value and the time derivative of $w_{\mathrm{DE}}$ today. According to this choice, the density of the DE fluid evolves as

$$
\rho_{\mathrm{DE}}(a)=3 m_{0}^{2} H_{0}^{2} \Omega_{\mathrm{DE}}^{0} a^{-3\left(1+w_{0}+w_{a}\right)} e^{-3 w_{a}(1-a)},
$$

where $\Omega_{\mathrm{DE}}^{0}$ is the density parameter of DE today.

For the functional forms of the EFT functions, we choose the following cases:

(i) Mla:

$$
\Omega(a)=\Omega_{0} a^{s_{0}}, \quad \gamma_{i}(a)=0,
$$

where $\left\{s_{0}, \Omega_{0}\right\}$ are the constant parameters defining the $\Omega$ function.

(ii) $M 1 b$ :

$$
\Omega(a)=\Omega_{0} a^{s_{0}}, \quad \gamma_{i}(a)=\gamma_{i}^{0} a^{s_{i}},
$$

where $\left\{s_{i}, \gamma_{i}^{0}\right\}$ are the parameters defining $\gamma_{i}$, with $i=1,2$.

(iii) $M 2 a$ :

$$
\begin{aligned}
& \Omega(a)=\Omega_{0} a^{-3\left(1+w_{0}+w_{a}\right)} e^{-3 w_{a}(1-a),} \\
& \gamma_{i}(a)=0,
\end{aligned}
$$

where $\Omega_{0}$ is a constant. This parametrization follows the DE density behavior, as shown in Eq. (7).

(iv) $M 2 b$ :

$$
\begin{aligned}
& \Omega(a)=\Omega_{0} a^{-3\left(1+w_{0}+w_{a}\right)} e^{-3 w_{a}(1-a),} \\
& \gamma_{i}(a)=\gamma_{i}^{0} a^{-3\left(1+w_{0}+w_{a}\right)} e^{-3 w_{a}(1-a),}
\end{aligned}
$$

where $\gamma_{i}^{0}(i=1,2)$ are constants.

We now focus on $\gamma_{1}$ and, in particular, on the its effects on the observables. As illustrated in the previous section, in the QS limit $\gamma_{1}$ does not appear in either $\mu$ or $\Sigma$; thus, it is

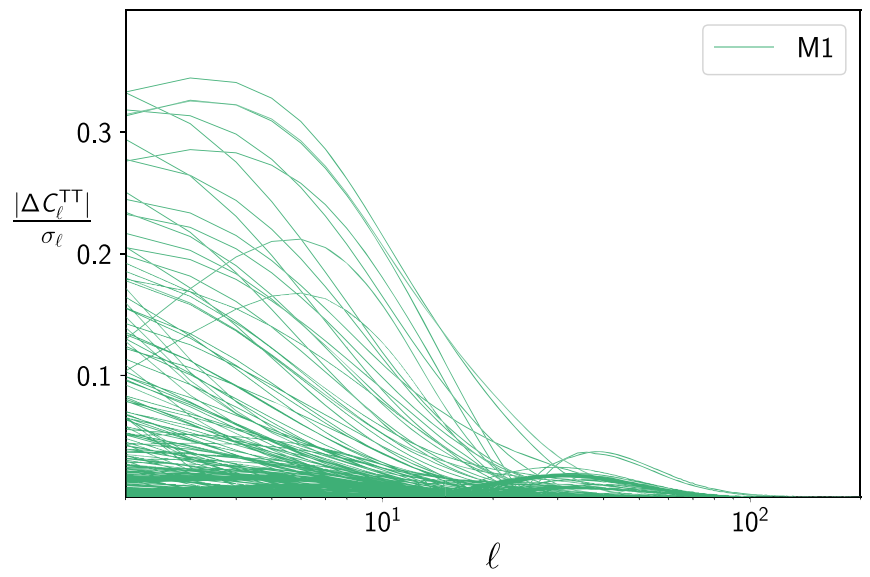

FIG. 1. Effects of $\gamma_{1}$ in $M 1$ on the TT power spectrum. We plot the deviation on the $C^{\mathrm{TT}}(\ell)$, in units of cosmic variance $\sigma_{\ell}=\sqrt{2 /(2 \ell+1)} C^{\mathrm{TT}}(\ell)$. We consider a sample of $\sim 10^{3}$ models, where both $\Omega$ and $\gamma_{1}$ are parametrized as in $M 1$. Here, $\Delta C^{\mathrm{TT}}$ is obtained as the difference between the model with $\Omega+\gamma_{1}$ and the one with solely $\Omega$.

hard to know a priori which role it plays at the perturbation level. In Ref. [49], in the context of the $\alpha$-basis, it has been shown that the kinetic function $\alpha_{K}$, when parametrized as a function of the DE density parameter on a $\Lambda \mathrm{CDM}$ background, is hard to constrain with cosmological data. We thus expect a similar result for $\gamma_{1}$ since the two functions are related [17].

For our study we consider the Mla model, and then we solely add $\gamma_{1}$, parametrized as in Eq. (9). We compute the difference $\Delta C^{\mathrm{TT}}(\ell)$ between the temperature-temperature power spectra for the two models, in units of cosmic variance $\sigma_{\ell}=\sqrt{2 /(2 \ell+1)} C^{\mathrm{TT}}(\ell)$, where for the latter $C^{\mathrm{TT}}(\ell)$ is the power spectra of the model with $\gamma_{1}=0$. We perform such a procedure for a sample of $\sim 10^{3}$ models, and we plot the results in Fig. 1. In such a sample, we have varied the background parameters in the ranges $w_{0} \in[-1.5,0], w_{a} \in[-1,0.5]$, and the EFT functions parameters $\Omega_{0} \in[0,3], \quad s_{0} \in[0,3], \quad \gamma_{1}^{0} \in[0,3], \quad$ and $s_{1} \in[-3,3]$. Let us note that these ranges have been chosen by requiring the viability of the model against ghost and gradient instabilities [17,50-57].

Analogously, in Fig. 2, we plot the deviations in $C^{\mathrm{TT}}(\ell)$ when both $\Omega$ and $\gamma_{1}$ are parametrized as in $M 2$, Eq. (11), considering the combinations $\left\{\Omega, \gamma_{1}=0\right\}$ and $\left\{\Omega, \gamma_{1}\right\}$. In this case, we consider a similar sample of $\sim 10^{3}$ models, where $w_{0}, w_{a}, \Omega_{0}$, and $\gamma_{1}^{0}$ are varied in the same ranges as in the previous case.

From Figs. 1 and 2, we can infer that the effects of $\gamma_{1}$ on the TT power spectrum become significant for $\ell \lesssim 100$, due to the late-time ISW effect. However, such contributions are always within the cosmic variance limit: We find that they never exceed $40 \%$ and $90 \%$ of comic variance for $M 1$ and $M 2$, respectively. For this reason, we conclude that 


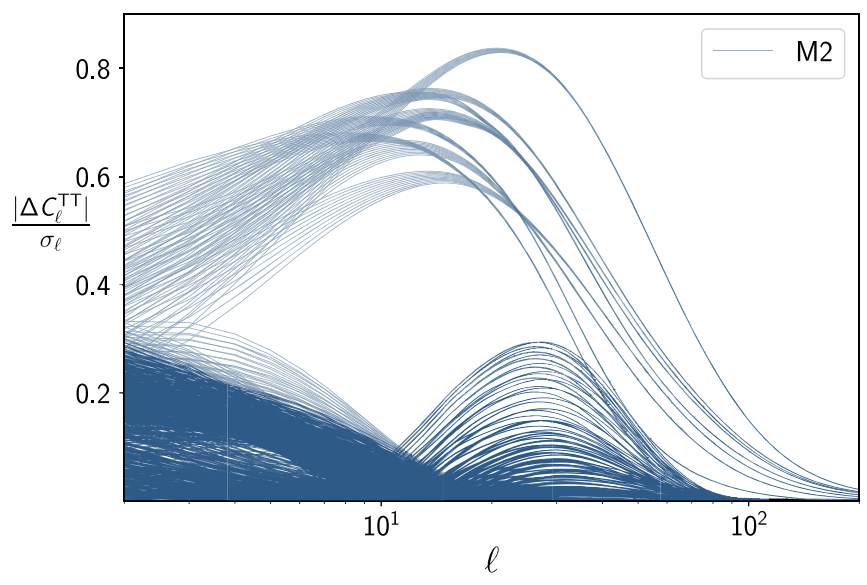

FIG. 2. Effects of $\gamma_{1}$ in $M 2$ on the TT power spectrum. We plot the deviation on the $C^{\mathrm{TT}}(\ell)$, in units of cosmic variance $\sigma_{\ell}=\sqrt{2 /(2 \ell+1)} C^{\mathrm{TT}}(\ell)$. We consider a sample of $\sim 10^{3}$ models, where both $\Omega$ and $\gamma_{1}$ are parametrized as in $M 2$. Note that $\Delta C^{\mathrm{TT}}$ is obtained as the difference between the model with $\Omega+\gamma_{1}$ and the one with solely $\Omega$.

it is unlikely that present surveys can constrain $\gamma_{1}$ or even that next-generation experiments will gain constraining power on such an operator. We show the results for the TT power spectrum, while we check that, for other observables, we get similar results. Nevertheless, $\gamma_{1}$ still plays an important role in the stability criteria of Horndeski theories. This means that, even if it does not directly modify the cosmological observables in a sizable way, $\gamma_{1}$ has a strong effect on the allowed parameter space for the other EFT functions (see Refs. [49,58] for the analogous case of $\alpha_{K}$ ). In particular, it enters in the condition for the avoidance of ghosts in the scalar sector [55].

As an illustrative example of the relevance of $\gamma_{1}$ in the stability, we consider the model described solely by $\gamma_{1}$ $\left(\left\{\Omega, \gamma_{2}\right\}=0\right)$, when $\gamma_{1}$ is parametrized as in Eq. (11) on a CPL background. We show in Fig. 3 how drastically $\gamma_{1}$ changes the stable $w_{0}-w_{a}$ parameter space, for different values of $\gamma_{1}^{0}$. We see that changing the value of the latter parameter has a clear impact on the stability of the CPL parameters: A positive value enlarges the stable parameter space, while a negative $\gamma_{1}^{0}$ shrinks it. Thus, we conclude that although $\gamma_{1}$ does not give any sizable effect on the observables, it cannot be neglected from the cosmological analysis because of its important role in the stability conditions. Moreover, as already pointed out in Sec. II A, when $\gamma_{2} \neq 0$ it immediately follows that $\gamma_{1} \neq 0$. For this reason, it is worth including such EFT functions in the present cosmological analysis.

\section{B. Codes and data sets}

For the present analysis, we employ the EFTCAMB/ EFTCOSMOMC codes $[46,59,60] .{ }^{1}$ The reliability of EFTCAMB has been tested against several Einstein-

\footnotetext{
${ }^{1}$ See http://www.eftcamb.org.
}

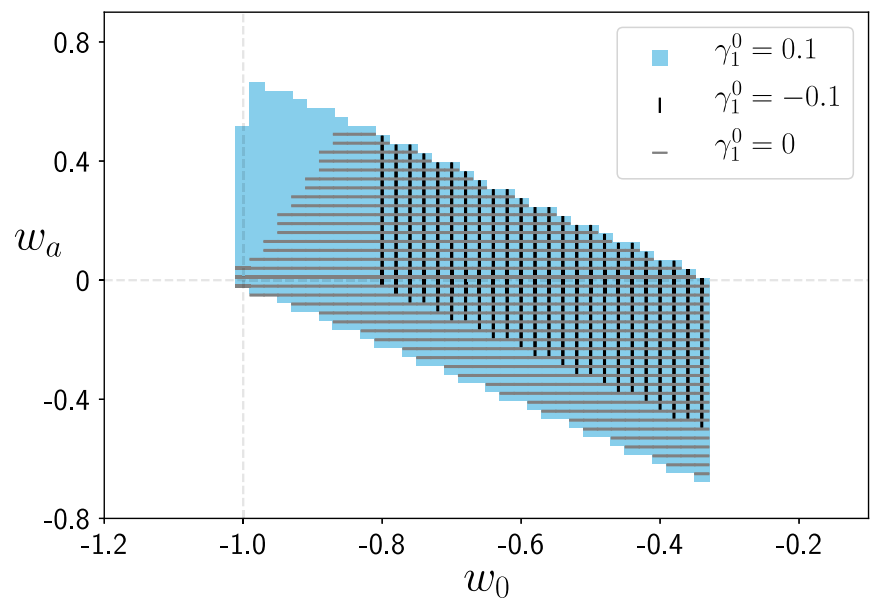

FIG. 3. Effects of $\gamma_{1}$ on the stable CPL parameter space. We consider the parametrization of $\gamma_{1}$ defined in Eq. (11) and compute the parameter space allowed by stability conditions, for different values of $\gamma_{1}^{0}$. The blue region represents the stable parameter space when $\gamma_{1}^{0}=0.1$, the horizontal grey lines refer to the case $\gamma_{1}^{0}=0$, and the vertical black lines to $\gamma_{1}^{0}=-0.1$.

Boltzmann solvers, and the agreement reaches the subpercent level [61].

We analyze Planck measurements [62,63] of CMB temperature on large angular scales, i.e., $\ell<29$ (low- $\ell$ likelihood), the $\mathrm{CMB}$ temperature on smaller angular scales, $30<\ell<2508$ (PLIK TT likelihood), and the CMB lensing map [64]. We also include baryonic acoustic oscillation (BAO) measurements from BOSS DR12 (consensus release) [65], local measurement of $H_{0}$ [66], and supernovae (SN) data from the Joint Light-curve Analysis "JLA" SN sample [67]. Along with the former data set, we consider measurements from weak gravitational lensing from the Kilo Degree Survey (KiDS) Collaboration [68-70]. In this case, we make a cut at nonlinear scales, by following the prescription in Refs. [71,72]. Practically, one performs a cut in the radial direction $k \leq 1.5 \mathrm{~h} \mathrm{Mpc}^{-1}$, and one removes the contribution from the $\xi^{-}$correlation function. In this way, the analysis has been shown to be sensitive only to the linear scales [72].

We list the flat priors used for the model parameters presented in the previous section: $w_{0} \in[-5,0], w_{a} \in$ $[-2,4]$ and $\left\{\Omega_{0}, s, \gamma_{1}^{0}, s_{1}, \gamma_{2}^{0}, s_{2}\right\} \in[-10,10]$.

\section{Forecast analysis}

We use the Fisher matrix approach [73-75], which is an inexpensive way of approximating the curvature of the likelihood at the peak, under the assumption that it is a Gaussian function of the model parameters. The main cosmological observables of next-generation galaxy redshift surveys, such as Euclid ${ }^{2}$ [76,77], DESI ${ }^{3}$ [78,79],

\footnotetext{
${ }^{2}$ See http://www.euclid-ec.org.

${ }^{3}$ See https://www.desi.lbl.gov.
} 
$\operatorname{LSST}^{4}[80]$, and SKA ${ }^{5}$ [81-84], are galaxy clustering (GC) and weak lensing (WL). WL can be measured with photometric redshifts and galaxy shape (ellipticity) data, while GC needs the position of galaxies in the sky and their redshifts to yield a three-dimensional map of the large-scale structure of the Universe. Though photometric GC can also give us some complementary information, especially in cross-correlation with WL, we use here only the more precise spectroscopic GC probe, which we assume to be independent of WL observables. This is a rather conservative approach, meaning that our constraints might be weaker than in the full case with cross-correlations, as it has been shown with present surveys such as DES [85]. Moreover, we do not have a generally valid approach to calculate the nonlinear matter power spectrum for models within the EFT formalism; thus, we cannot include nonlinear scales in our modeling of the Fisher matrix. Therefore, we need to limit ourselves to linear scales, which might yield to large forecasted errors, especially for the WL analysis which is very sensitive to nonlinearities. In practice, the largest scales we take into account correspond to $k_{\min }=0.0079 \mathrm{~h} / \mathrm{Mpc}^{-1}$ and, since we want to restrict ourselves to linear scales, we use a hard cutoff at $k_{\max }=0.15 \mathrm{~h} / \mathrm{Mpc}^{-1}$ and at a maximum multipole of $\ell_{\max }=1000$. Finally, we perform the forecast analysis only for the cases without massive neutrinos for the following reasons: First, we cut our analysis at nonlinear scales, and this is the regime where the larger effects coming from the presence of the neutrinos are expected; second, the results we get from cosmological data show that massive neutrinos do not considerably affect the constraints (see Sec. IV).

\section{Galaxy clustering}

In order to compute the predictions for galaxy clustering, we need to compute $P_{\text {obs }}$, which is the Fourier transform of the two-point correlation function of galaxy number counts in redshift space. ${ }^{6}$ The observed galaxy power spectrum follows the matter power spectrum of the underlying dark matter distribution $P(k)$ up to a bias factor $b(z)$ and some effects related to the transformation from configuration space into redshift space. We assume the galaxy bias to be local and scale independent, though modified gravity theories might, in general, predict a scale dependence [89]. To write down the observed power spectrum, we neglect other relativistic and nonlinear corrections, and we follow Ref. [74], so that we end up with

\footnotetext{
${ }^{4}$ See https://www.lsst.org.

${ }^{5}$ See https://www.skatelescope.org.

${ }^{6}$ It has been shown in previous works that a Fisher matrix forecast analysis which makes use of the full shape of the $P_{\text {obs }}$ allows us to obtain very similar constraints to those obtained by using the multipole method [86-88].
}

$P_{\mathrm{obs}}(k, \tilde{\mu}, z)=\frac{D_{A, f}^{2}(z) H(z)}{D_{A}^{2}(z) H_{f}(z)} B^{2}(z) e^{-k^{2} \tilde{\mu}^{2} \sigma_{\mathrm{tot}}^{2}} P(k, z)$,

with

$$
\sigma_{\mathrm{tot}}^{2}=\sigma_{r}^{2}+\sigma_{v}^{2}, \quad B(z)=b(z)\left(1+\beta_{d}(z) \tilde{\mu}^{2}\right),
$$

where $B(z)$ contains the so-called Kaiser effect [90,91], $\beta_{d}(z) \equiv f(z) / b(z)$, and $f \equiv d \ln G / d \ln a$ is the linear growth rate of matter perturbations. In this equation, $\tilde{\mu}$ is the cosine of the angle between the line of sight and the 3D wave vector $\vec{k}$. Every quantity in this equation depends on all cosmological parameters and is varied accordingly, except for those with a subscript $f$, which denote an evaluation at the fiducial value. In particular, we marginalize over the galaxy bias parameter for each redshift bin. Here, $D_{A}(z)$ is the angular diameter distance, and the exponential factor represents a damping term with $\sigma_{r}^{2}+\sigma_{v}^{2}$, where $\sigma_{r}$ is the error induced by spectroscopic redshift measurements and $\sigma_{v}$ is the velocity dispersion associated with the Finger of God effect [74]. We marginalize over this last parameter [92] and take a fiducial value $\sigma_{v}=$ $300 \mathrm{~km} / \mathrm{s}$ compatible with the estimates in Ref. [93]. See Refs. [74,94,95] for further details.

The Fisher matrix is then computed by taking derivatives of $P_{\text {obs }}$ with respect to the cosmological parameters and by integrating these together with a Gaussian covariance matrix and a volume term, over all angles and all scales of interest $[94,96]$.

The galaxy number density $n(z)$ we use here peaks at a redshift of $z=0.75$, and it is similar to the spectroscopic DESI-ELG survey found in [78]. We also use their expected redshift errors and bias specifications, but a slightly larger area of 15000 square degrees. Such specifications will allow us to make predictions on cosmological and model parameters which can soon be compared with real data. Let us note that using specifications closer to the SKA-2 survey would probably result in stronger constraints than those we will obtain with DESI-like specifications. However, data from SKA-2 will not be available in the next decade. In this regards, our results can provide a better insight on the constraining power of a near future survey on MG theories.

\section{Weak lensing}

Weak lensing is the measurement of cosmic shear, which represents the ellipticity distortions in the shapes of galaxy images. This in turn is related to deflection of light due to the presence of matter in the Universe. Therefore, WL is a very powerful probe of the distribution of large-scale structures, and due to its tomographic approach, it provides valuable information about the accelerated expansion of the Universe. Assuming small gravitational potentials and large separations, we can link cosmic shear to the matter power spectrum, giving direct constraints on the cosmological 
parameters. In this case, we use tomographic WL in which we measure the cosmic shear in a number of wide redshift bins, given by a window function $W_{i}(z)$ at the bin $i$, which is correlated with another redshift bin $j$. The width of these window functions depends on a combination of the photometric redshift errors and the galaxy number densities. The cosmic shear power spectrum can thus be written as a matrix with indices $i, j$, namely,

$$
C_{i j}(\ell)=\frac{9}{4} \int_{0}^{\infty} \mathrm{d} z \frac{W_{i}(z) W_{j}(z) H^{3}(z) \Omega_{m}^{2}(z)}{(1+z)^{4}} \Sigma^{2}(k, z) P_{m},
$$

with $P_{m}$ evaluated at the scale $\ell / r(z)$, where the comoving distance is $r(z)$. In modified gravity, the lensing equation is modified by the term $\Sigma$ in Eq. (4); thus, it turns out that such a term also appears in the evaluation of the power spectrum. For the Fisher matrix, we follow the same procedure as in Refs. [39,94], where for the actual unconvoluted galaxy distribution function, we have assumed

TABLE I. The $2 \sigma$ marginalized constraints on cosmological parameters. These values are obtained through the analysis of the full data set presented in Sec. III B.

\begin{tabular}{lccccc}
\hline \hline Model & $10^{9} A_{s}$ & $n_{s}$ & $\Omega_{m}$ & $H_{0}$ & $\Sigma m_{\nu}$ \\
\hline$\Lambda \mathrm{CDM}$ & $2.11_{-0.12}^{+0.12}$ & $0.969_{-0.009}^{+0.009}$ & $0.297_{-0.013}^{+0.013}$ & $68.7_{-1.0}^{+1.1}$ & \\
$\Lambda \mathrm{CDM}+\nu$ & $2.22_{-0.19}^{+0.23}$ & $0.974_{-0.011}^{+0.012}$ & $0.300_{-0.014}^{+0.015}$ & $68.4_{-1.2}^{+1.2}$ & $<0.288$ \\
M1a & $2.21_{-0.21}^{+0.21}$ & $0.974_{-0.012}^{+0.012}$ & $0.295_{-0.016}^{+0.017}$ & $68.7_{-1.7}^{+1.8}$ & \\
M1a $+\nu$ & $2.29_{-0.22}^{+0.25}$ & $0.976_{-0.013}^{+0.013}$ & $0.298_{-0.018}^{+0.017}$ & $68.4_{-1.6}^{+1.8}$ & $<0.281$ \\
M1b & $2.19_{-0.23}^{+0.24}$ & $0.973_{-0.012}^{+0.013}$ & $0.293_{-0.017}^{+0.017}$ & $68.9_{-1.8}^{+1.8}$ & \\
M1b $+\nu$ & $2.28_{-0.25}^{+0.25}$ & $0.975_{-0.015}^{+0.013}$ & $0.295_{-0.016}^{+0.018}$ & $68.8_{-1.7}^{+1.8}$ & $<0.347$ \\
M2a & $2.27_{-0.20}^{+0.21}$ & $0.972_{-0.010}^{+0.010}$ & $0.302_{-0.014}^{+0.015}$ & $68.1_{-1.4}^{+1.3}$ & \\
M2a $+\nu$ & $2.35_{-0.22}^{+0.24}$ & $0.975_{-0.011}^{+0.011}$ & $0.303_{-0.014}^{+0.016}$ & $67.9_{-1.4}^{+1.3}$ & $<0.236$ \\
M2b & $2.20_{-0.26}^{+0.28}$ & $0.968_{-0.013}^{+0.013}$ & $0.300_{-0.016}^{+0.016}$ & $68.6_{-1.6}^{+1.8}$ & \\
M2b $+\nu$ & $2.30_{-0.29}^{+0.29}$ & $0.970_{-0.014}^{+0.014}$ & $0.304_{-0.017}^{+0.017}$ & $68.5_{-1.6}^{+1.7}$ & $<0.543$ \\
\hline \hline
\end{tabular}

$$
n(z) \propto\left(z / z_{0}\right)^{2} \exp \left(-\left(z / z_{0}\right)^{3 / 2}\right),
$$

and SKA-like specifications for WL [97]. Although these are rather futuristic specifications, we choose them in order to improve our WL constraints, which otherwise would not be very informative since we consider only linear scales.

\section{RESULTS}

In addition to the mentioned variety of gravity models, we also consider two different cosmological scenarios: one with massless neutrinos and the other with a massive neutrino component. In Table I, we show the results for the cosmological parameters for the models $M 1 a / b, M 2 a / b$, with and without massive neutrinos. In the same table, we added, for comparison, the $\Lambda \mathrm{CDM}$ results. In Table II, we show the constraints on the corresponding model parameters.

We also studied the effects of giving different hierarchies to the massive neutrino species, considering the normal $(\mathrm{NH})$, inverted $(\mathrm{IH})$, and degenerate $(\mathrm{DH})$ hierarchy scenarios. The impact of different hierarchies on cosmological constraints was first considered both in $\Lambda \mathrm{CDM}$ [98-100] and alternative cosmologies [101,102], and it is expected that the probability of breaking the degeneracy between them increases as the bound on the total mass of neutrinos becomes tighter [98]. Nevertheless, we find that such different scenarios are indistinguishable when using this combination of data. The reason can be found in the following argument: In order to get any insight on a preferred hierarchy, one should get a sensitivity on the sum of neutrino masses of $\Sigma m_{\nu}<0.2 \mathrm{eV}$ at $2 \sigma$; in particular, to exclude the IH, it has to be $\Sigma m_{\nu}<0.1 \mathrm{eV}$, as discussed in Ref. [98]. For the data sets and models considered in the present work, $\Sigma m_{\nu}$ never goes below this threshold at $2 \sigma$ (see Table I).

We find that, regardless of the model considered, the cosmological parameters $\left\{A_{s}, n_{s}, H_{0}, \Omega_{m}, \Sigma m_{\nu}\right\}$ are all consistent with the $\Lambda \mathrm{CDM}$ scenario at $2 \sigma$. Furthermore, we do not find relevant differences when considering different combinations of the data sets; for such a reason,

TABLE II. The $2 \sigma$ marginalized constraints on model parameters. These values are obtained through the analysis of the full data set

\begin{tabular}{|c|c|c|c|c|c|c|c|c|}
\hline Model & $w_{0}$ & $w_{a}$ & $\Omega_{0}$ & $s_{0}$ & $\gamma_{1}^{0}$ & $s_{1}$ & $\gamma_{2}^{0}$ & $s_{2}$ \\
\hline M1a & $-1.04_{-0.16}^{+0.14}$ & $0.22_{-0.39}^{+0.46}$ & $-0.07_{-0.18}^{+0.17}$ & $>0.435$ & & & & \\
\hline $\mathrm{M} 1 \mathrm{a}+\nu$ & $-1.02_{-0.18}^{+0.13}$ & $0.12_{-0.37}^{+0.49}$ & $-0.04_{-0.21}^{+0.15}$ & $>0.240$ & & & & \\
\hline M1b & $-1.07_{-0.16}^{+0.15}$ & $0.30_{-0.42}^{+0.47}$ & $0.03_{-0.25}^{+0.31}$ & $>0.215$ & $>0.217$ & -- & $-0.9_{-2.0}^{+1.3}$ & $>0.330$ \\
\hline $\mathrm{M} 1 \mathrm{~b}+\nu$ & $-1.08_{-0.15}^{+0.16}$ & $0.24_{-0.48}^{+0.49}$ & $0.01_{-0.33}^{+0.33}$ & $>0.296$ & $>0.103$ & -- & $-1.9_{-5.0}^{+2.3}$ & $>0.147$ \\
\hline M2a & $-0.946_{-0.060}^{+0.090}$ & $-0.098_{-0.28}^{+0.25}$ & $0.018_{-0.019}^{+0.032}$ & & & & & \\
\hline $\mathrm{M} 2 \mathrm{a}+\nu$ & $-0.950_{-0.056}^{+0.087}$ & $-0.11_{-0.30}^{+0.23}$ & $0.019_{-0.020}^{+0.037}$ & & & & & \\
\hline $\mathrm{M} 2 \mathrm{~b}$ & $-0.94_{-0.13}^{+0.15}$ & $-0.31_{-0.63}^{+0.48}$ & $0.047_{-0.051}^{+0.068}$ & & $>0.295$ & & $-0.23_{-0.32}^{+0.26}$ & \\
\hline $\mathrm{M} 2 \mathrm{~b}+\nu$ & $-0.93_{-0.14}^{+0.15}$ & $-0.61_{-0.66}^{+0.66}$ & $0.080_{-0.081}^{+0.099}$ & & $>0.151$ & & $-0.36_{-0.47}^{+0.36}$ & \\
\hline
\end{tabular}
presented in Sec. III B. Here, -- means that the parameter is left unconstrained. 
TABLE III. Forecasted $2 \sigma$ errors on the cosmological parameters for a next-generation spectroscopic galaxy clustering measurement plus a photometric weak lensing experiment, using Planck priors.

\begin{tabular}{lcccc}
\hline \hline Model & $2 \sigma\left(10^{9} A_{s}\right)$ & $2 \sigma\left(\Omega_{m}\right)$ & $2 \sigma\left(H_{0}\right)$ & $2 \sigma\left(n_{s}\right)$ \\
\hline M1a & $4.0 \%$ & $1.9 \%$ & $1.0 \%$ & $0.8 \%$ \\
M1b & $4.2 \%$ & $2.2 \%$ & $1.1 \%$ & $0.9 \%$ \\
M2a & $0.02 \%$ & $1.4 \%$ & $0.8 \%$ & $0.7 \%$ \\
M2b & $4.4 \%$ & $1.7 \%$ & $1.0 \%$ & $0.8 \%$ \\
\hline \hline
\end{tabular}

TABLE IV. Forecasted $2 \sigma$ marginalized constraints on model parameters. These values are obtained with the combination of $\mathrm{GC}+\mathrm{WL}+$ Planck for a future next-generation galaxy survey.

\begin{tabular}{lcccccc}
\hline \hline Model & $2 \sigma\left(w_{0}\right)$ & $2 \sigma\left(w_{a}\right)$ & $2 \sigma\left(\Omega_{0}\right)$ & $2 \sigma\left(s_{0}\right)$ & $2 \sigma\left(\gamma_{2}^{0}\right)$ & $2 \sigma\left(s_{2}\right)$ \\
\hline M1a & $2.0 \%$ & $50 \%$ & $110 \%$ & $68 \%$ & - & - \\
M1b & $2.2 \%$ & $40 \%$ & $128 \%$ & $96 \%$ & $240 \%$ & $136 \%$ \\
M2a & $1.9 \%$ & $44 \%$ & $22 \%$ & - & - & - \\
M2b & $2.6 \%$ & $18 \%$ & $48 \%$ & - & $40 \%$ & - \\
\hline \hline
\end{tabular}

we only show the results for the full data set analysis. Such constraints are not considerably affected by the presence of massive neutrinos or by the modifications to gravity introduced through $\Omega, \gamma_{1}$, and $\gamma_{2}$. Finally, as shown in Table II, $\gamma_{1}$ is really weakly constrained by the data, and the results are mostly compatible with the prior we used. The cut in the negative prior range is due to the requirement of avoiding ghost instability which enforces a positive $\gamma_{1}^{0}$. The same happens for the exponent parameter $s_{1}$ which is left totally unconstrained. This result is expected and in line with the discussion presented in Sec. III A.

Let us now move to the forecasts. We use the bestfit parameters of the corresponding model from Tables III and IV as fiducial values for the following reasons: (1) To avoid unconstrained parameters. In the $M 1$ case, the $\Lambda \mathrm{CDM}$ limit corresponds to $\Omega_{0}=\gamma_{i}^{0}=0$. Using these values would distort the results on the exponentials $s_{i}$, which then will be unconstrained. Such results would depend only on the choice of the fiducial values and will not be informative on the constraining power of the nextgeneration-like survey. (2) As can be noticed from Table II, we obtained lower limit bounds on the $\gamma_{1}^{0}$ parameter. The value expected for $\gamma_{1}^{0}$ in the $\Lambda \mathrm{CDM}$ limit is 0 , and it is excluded by our constraints. Thus, we deduced that the best-fit parameters are more representative as fiducial values for these models than those of $\Lambda \mathrm{CDM}$. For the models with $\gamma_{1}$, we have used $\gamma_{1}^{0}=5.0$ and $s_{1}=1.4$ for $M 1 b$, while for $M 2 b$ we used $\gamma_{1}^{0}=4.4$. We considered these values as fixed since we proved that the effect of $\gamma_{1}$ is negligible on the cosmological observables, even for nextgeneration surveys. Let us stress that the results we obtain stay as long as the present best-fit values remain.

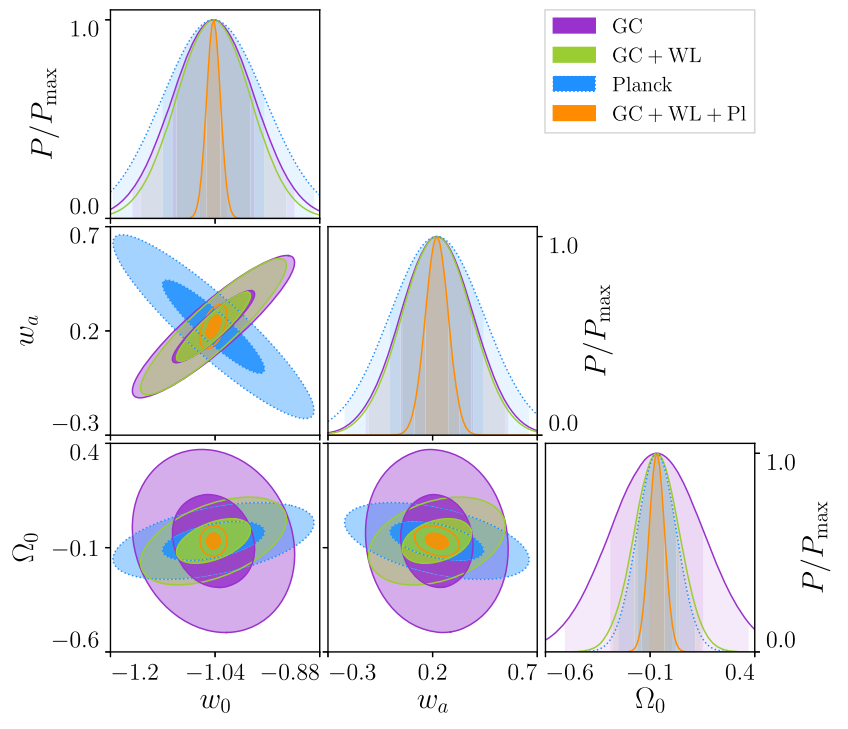

FIG. 4. Forecast for model $M 1 a$ for the equation of state parameters $w_{0}, w_{a}$ and the model parameter $\Omega_{0}$. In purple, we have GC, in green GC + WL (assuming they are independent), in blue the Planck prior from our MCMC, and in orange the combination of GC $+\mathrm{WL}+$ Planck.

In Fig. 4, we show the forecasted $1 \sigma$ and $2 \sigma$ constraints, for the model parameters of Mla, for different combinations of the next-generation data sets. From such plots, we can see the effect of the different data sets: We find a common feature in the $w_{0}-w_{a}$ plane, where the $\mathrm{GC}$ analysis removes the degeneracy coming from the Planck measurements; analogously, we can appreciate how the inclusion of $\mathrm{WL}$ in the CG analysis considerably increases the constraints on $\Omega_{0}$.

In Figs. 5 and 6, we compare the forecasted marginalized distribution for the models $M 1 b-M 2 b$ and $M 1 a-M 2 a$, respectively, obtained through the analysis with the full $\mathrm{CG}+\mathrm{WL}+$ Planck data set. From these results, we can see that the $M 1 b-M 2 b$ models have the fiducial values of $\Omega_{0}$ compatible within the error bars, while in the $w_{0}-w_{a}$ parameter space, the models could be distinguished at more than $5 \sigma$. Alternatively, in the M1a-M2a comparison plot, while the constraints on cosmological parameters, $H_{0}$ and $\Omega_{m}$, are very similar, the constraint on $\Omega_{0}$ for the model $M 2 a$ is much stronger (GC and Planck). This is due to the fact that in $M 2 a$ the parameter $\Omega_{0}$ is related to $w_{0}-w_{a}$ and therefore can be measured indirectly by measuring the equation of state of dark energy. In the marginal likelihood of $\Omega_{0}$, both models could be distinguished at almost the $3 \sigma$ level.

In Tables III and IV, we list the forecasted $2 \sigma$ errors, respectively, on the cosmological and model parameters obtained with the GC $+\mathrm{WL}+$ Planck combination, for a future next-generation galaxy survey. Compared to present data, we find that future surveys, in general, will slightly improve the constraint on cosmological parameters; 


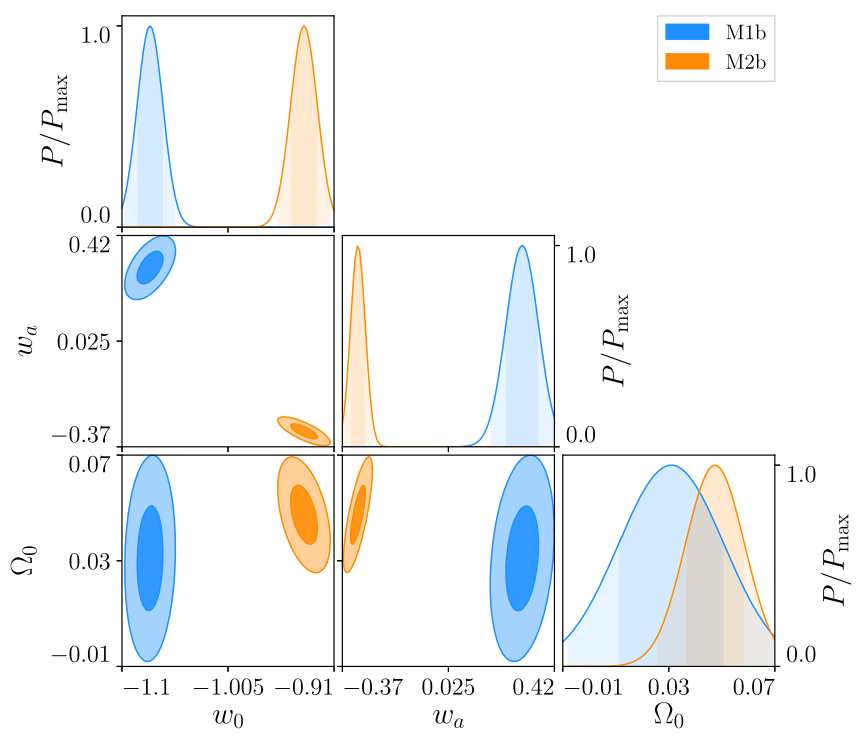

FIG. 5. Forecasts comparing models $M 1 b$ (blue) and $M 2 b$ (orange) for the equation of state parameters $w_{0}, w_{a}$ and the model parameter $\Omega_{0}$. Both Fisher matrices are computed for the combined GC $+\mathrm{WL}+$ Planck case.

notably, for the $A_{s}$ parameter in $M 2 a$, the error reduces by 2 orders of magnitude in the forecasts. Such an improvement is due to the WL which breaks the degeneracy with $\mathrm{CG}$ and Planck. Furthermore, future surveys will improve the constraints on the model parameters by 1 order of magnitude. Even better, they will set constraints of order $\lesssim 100 \%$ on $s_{0}, s_{2}$ parameters for which the present data adopted in this work are only able to set lower bounds.

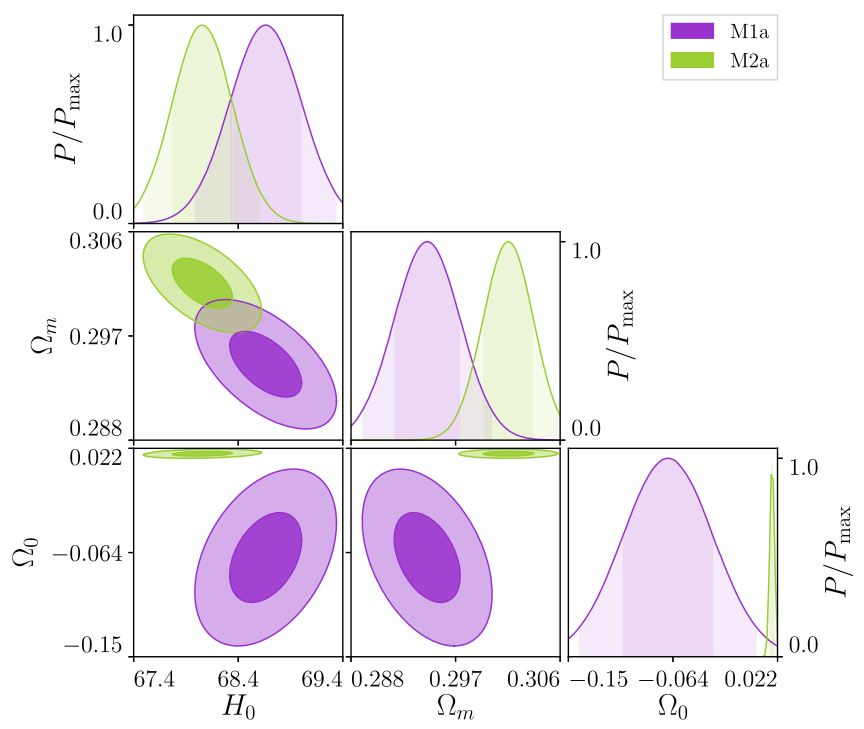

FIG. 6. Forecasts comparing models M1a (purple) and M2a (green) for the model parameter $\Omega_{0}$ and the cosmological parameters $H_{0}$ and $\Omega_{m}$. Both Fisher matrices in this plot are computed for the combined GC $+\mathrm{WL}+$ Planck case.
We also explore the deviations from GR of the $\mu$ and $\Sigma$ functions, and we test the goodness of their QS approximations. For these purposes, we compare the QS expressions for $\mu$ and $\Sigma$, as reported in Eq. (5), with those obtained by using their exact expressions as in Eq. (4) (hereafter, we will use the superscript "ex"). These are computed by evolving the full dynamics of perturbations with EFTCAMB. Finally, we show the deviations of the exact solutions with respect to GR. The cosmological/model parameters are chosen according to the best-fit values in Tables I and II. We did not include the case of massive neutrinos since their presence does not make any consistent difference.

For the Mla/b models, we find that the QS approximation is a valid assumption at the values of $z$ and $k$ considered, being the difference between $\mu / \Sigma$ QS and exact $\sim 10^{-3}(0.1 \%)$, and $\mu / \Sigma$ are also compatible with GR $\left(|\mu-1|\right.$ and $\left.|\Sigma-1| \sim 10^{-3}\right)$.

For the $M 2 a / b$ cases, we find different results, as we show in Fig. 7. In the top panels, we plot the difference between the QS and exact solutions. We can see that the QS approximation is a valid assumption within the sound horizon $\left(k_{s}=c_{s} k_{H}\right.$, black line). Indeed, for both $M 2 a$ and $M 2 b$, the quantity $\Delta \Sigma=\left|\Sigma^{\mathrm{ex}}-\Sigma^{\mathrm{QS}}\right|$ reaches around $0.1 \%$ deep inside the $k_{s}$, while outside, it grows to a few
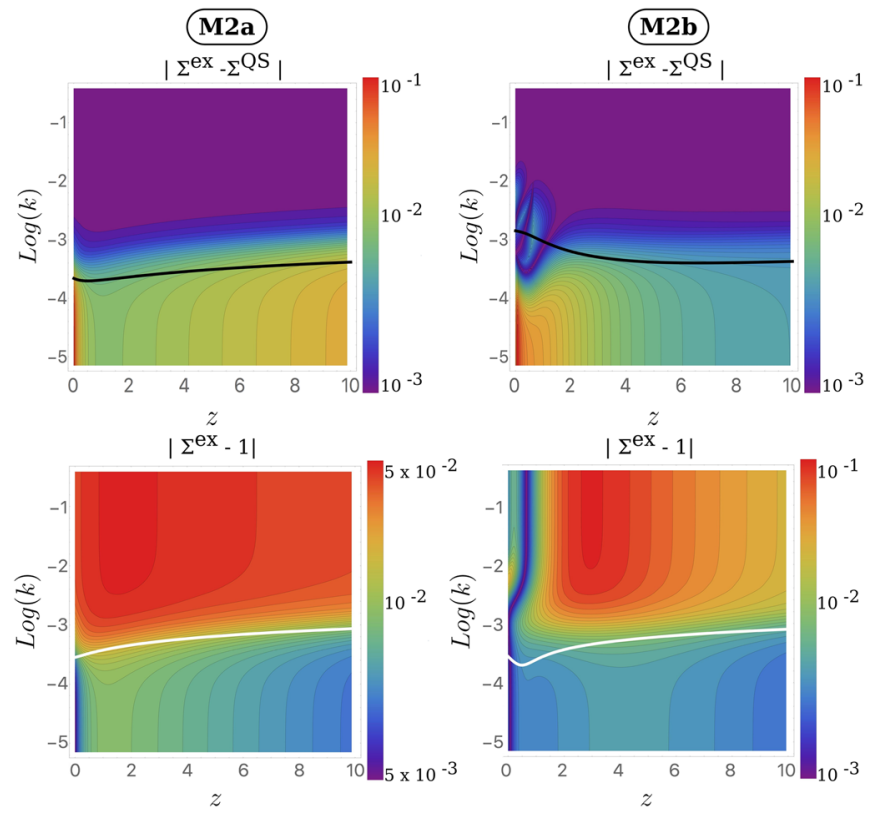

FIG. 7. Top panels: Absolute relative differences between the exact ("ex") and QS calculations of $\Sigma$ for the $M 2 a$ model (top left) and the $M 2 b$ model (top right). The black line corresponds to the dark energy sound horizon scale. Bottom panels: Absolute relative differences between exact $\Sigma$ in models $M 2 a$ (bottom left) and $M 2 b$ (bottom right) and the general relativity value. The white line corresponds to the Compton scale associated with the extra scalar DOF. In all panels, we have used the best-fit values for the parameters in Tables I and II. For reference, we checked that such results show a behavior similar to what we obtain for the $\mu$ function. 
percent, reaching around $10 \%$ at small $z$. Finally, we explore the deviations of the $M 2$ model from GR. From the bottom panels in Fig. 7, one can clearly see that the Compton wavelength $\left(k_{C}\right.$, white line) associated with the extra scalar DOF actually introduces a transition between two regimes. In fact, the large deviations from GR at $k>k_{C}$ can reach 5\% at all redshifts $(M 2 a)$ or for $z>1(M 2 b)$. On the other hand, at larger scales $\left(k<k_{C}\right), \Sigma$ gets closer to its GR value, with a relative difference which is always below $1 \%$.

Such results are particularly interesting when we want to extend the forecasts and analyze the constraining power of future surveys on the phenomenological functions $\Sigma, \mu$. Using the QS expressions in Eq. (5) and the Fisher matrices obtained for the model parameters, we can calculate a derived Fisher matrix $\tilde{\mathbf{F}}$ for the forecasted errors on the derived quantities $\mu$ and $\Sigma$ as follows:

$$
\tilde{\mathbf{F}}=\mathbf{J}^{T} \mathbf{F} \mathbf{J},
$$

with

$$
\mathbf{J} \equiv J_{i j}=\frac{\partial p_{i}}{\partial \tilde{q}_{j}},
$$

where $p_{i}$ is a vector containing all the parameters of the model [standard cosmological parameters $\left(\Omega_{m}, H_{0}, A_{s}, n_{s}\right.$, $w_{0}$ and $\left.w_{a}\right)$ together with EFT parameters $\left.\left(\Omega_{0}, \gamma_{2}^{0}, s_{i}, \ldots\right)\right]$ and $\tilde{q}$ is a vector containing the standard cosmological parameters plus $\mu$ and $\Sigma$. Through the QS limit, we can compute $\partial \tilde{q}_{j} / \partial p_{i}$ since we know the functions $\Sigma\left(k, z, p_{i}\right)$ and $\mu\left(k, z, p_{i}\right)$. So, in order to compute the Jacobian $\mathbf{J}$, it can be shown that its inverse is equal to $J_{i j}^{-1}=\partial \tilde{q}_{j} / \partial p_{i}$.

We compute the derived Fisher matrices at a fixed scale, i.e., $k=0.01 h / \mathrm{Mpc}$, which is well inside the Compton scale, for which the QS approximation is valid and where linear structure formation still holds. We do the same for six redshift bins, between $z=0.5$ and $z=2.0$, which cover typical redshift ranges of future surveys. We report in Fig. 8

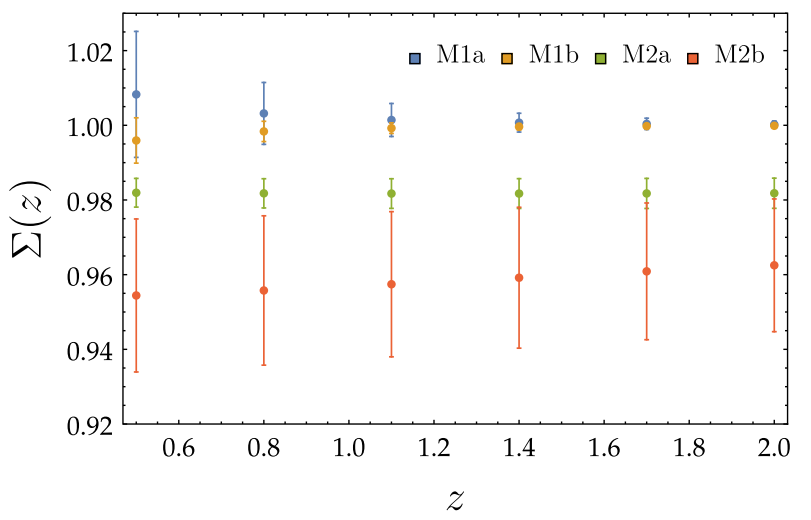

FIG. 8. Forecasted $2 \sigma$ errors on the $\Sigma(z)$ parameter, for all four models considered in this work. The errors turn out to be quite small since the parameter dependence of $\Sigma$ is very mild in the QS limit. We fix $k=0.01 h / \mathrm{Mpc}$. the $2 \sigma$ error on $\Sigma(z)$ after marginalizing over all other parameters. We obtain the same errors for $\mu(z)$ since, for our models, this function behaves extremely similarly to $\Sigma(z)$.

For models $M l a$ and $M 1 b$, the errors obtained are of the order of $10^{-3}$, decreasing towards $10^{-4}$ for higher redshifts $(z>1.5)$, since there the functions $\mu(z)$ and $\Sigma(z)$ asymptotically tend to 1 , independent of the cosmological parameters, which then implies very small predicted errors. For models $M 2 a$ and $M 2 b$, the forecasted errors are constant in redshift, approximately $4 \times 10^{-3}$ and $2 \times 10^{-1}$, respectively.

\section{CONCLUSION}

In this work, we have explored the phenomenology of the class of Horndeski theory compatible at all redshifts with the gravitational wave constraints, which we called surviving Horndeski (sH). For this class of modified gravity models, we have provided cosmological constraints from present-day and upcoming large-scale surveys. We performed the study by means of the EFT framework: Thus, we moved the problem of choosing the $\mathrm{sH}$ functions $\left\{\mathcal{K}, G_{3}, G_{4}\right\}$ to selecting the free functions in the EFT formalism $\left\{\Omega, \gamma_{1}, \gamma_{2}\right\}$. For this particular class of models, the mapping procedure becomes quite straightforward, and there exists a one-to-one correspondence between each EFT function and the Horndeski ones. Modeling the EFT functions instead of the $G_{i}$ functions could, in some cases, result in oversimplified descriptions of the evolution of the Universe, which might miss a significant signature of modified gravity [103], even though model-independent descriptions led to some relevant and novel predictions about modifications of gravity [45,57,104-108].

We found that the main contribution of the EFT function $\gamma_{1}$ dwells in the late-time ISW effect, but always within the cosmic variance limits. We could then infer that neither present nor future surveys can constrain the evolution of $\gamma_{1}$ : This is confirmed by the results of our cosmological analysis in Table II, which left the $\gamma_{1}$ parameters completely unconstrained. However, let us note that the use of sophisticated multitracer techniques could allow us to overcome the cosmic variance limitations [109]. Moreover, we showed that $\gamma_{1}$ still has an important role in defining the viable parameter space of the theory; thus, it cannot be neglected in the cosmological analysis.

We provided a constraint analysis of the $\mathrm{sH}$ models, using present-day data and forecasts from combinations of GC and WL for a generic next-generation galaxy survey. We found that future surveys will be able to increase the precision on the model parameter constraints by 1 order of magnitude. In the forecast analysis, we did not notice any peculiarity at the level of the cosmological parameters, whose error bars are compatible among all models; we highlighted many features related to the model parameters for the single cases. For example, we were able to show that 
the correlation between $\gamma_{2}^{0}$ and $w_{a}$ in $M 2 b$ translates into tighter constraints on the latter parameter. Furthermore, in Figs. 5 and 6 we showed the MIb/M2b and Mla/M2a model comparisons for the forecasted marginalized results. From such comparisons, we are able to state that, given these fiducials, we will be able to distinguish $M 1 b$ from $M 2 b$ at the $5 \sigma$ level in the $w_{0}-w_{a}$ parameter space and, analogously, Mla from $M 2 a$ at $3 \sigma$ in the marginal likelihood of $\Omega_{0}$.

We studied the deviations of $M 1$ and $M 2$, with respect to GR, in terms of the phenomenological functions $\mu$ and $\Sigma$. We found that $M 1$ is compatible with GR within $0.1 \%$, while $M 2 a / b$ show a $5 \%$ departure from GR, at scales smaller than the Compton scale. We then tested the validity of the QS approximation, and we found that it is a valid assumption for the $M 1$ model regardless of the scale, while, in the case of $M 2$, we numerically checked that the validity of the QS limit is deeply connected with the definition of the dark energy sound horizon scale: Within this scale, the approximation holds at the subpercent level, while it breaks down at larger scales. This result concretely proves what was found in [9]. Finally, we propagated the forecasted errors on the model parameters into $\mu$ and $\Sigma$, and we found that for models $M I a / b$ the forecasted $2 \sigma$ errors, despite being very small $\left(\sim 10^{-3}\right)$, will not be able to discriminate these models from GR at more than $1 \sigma$ because both $\mu, \Sigma$ are close to the GR values, i.e., $\mu=\Sigma=1$. On the contrary, for models $M 2 a / b$ the discrepancy from GR is large, and the errors are small enough, such that, provided the same bestfit values hold, we could distinguish these models from standard GR at more than $3 \sigma$ in the derived quantities $\mu$ and $\Sigma$, using future galaxy surveys combined with $\mathrm{CMB}$ priors.

We conclude that the surviving class of Horndeski theory offers an interesting cosmological phenomenology, even after the $c_{t}^{2}=1$ constraint, and it is worth further investigating with the upcoming observational data. Future surveys will provide a large amount of high precision data, not only limited to the galaxy clustering and weak lensing observables considered here, and the inclusion in the data analysis of a proper treatment of nonlinear scales will further improve their power in constraining [39]. Such high sensitivity will set tiny constraints on any signature of deviations from GR, allowing us to discriminate among gravity models, and it will represent the ultimate test for the $\Lambda \mathrm{CDM}$ scenario.

\section{ACKNOWLEDGMENTS}

We thank Martin Kilbinger, Martin Kunz, Matteo Martinelli, Shinji Mukohyama, Valeria Pettorino, and Alessandra Silvestri for useful discussions and comments on this work. The research of N.F. is supported by Fundação para a Ciência e a Tecnologia (FCT) through national funds (UID/FIS/04434/2013), by FEDER through COMPETE2020 (POCI-01-0145-FEDER-007672) and by FCT project "DarkRipple-Spacetime ripples in the dark gravitational Universe" with Ref. No. PTDC/FIS-OUT/ 29048/2017. S. P. acknowledges support from the NWO and the Dutch Ministry of Education, Culture and Science (OCW), and also from the D-ITP consortium, a program of the NWO that is funded by the OCW. S. C. acknowledges support from CNRS and CNES grants. N. F., S. C., and S. P. acknowledge the COST Action (CANTATA/CA15117), supported by COST (European Cooperation in Science and Technology). N. A. L. acknowledges support from DFG through the Project No. TRR33 "The Dark Universe" and would like to thank the Department of Physics of the University of Lisbon for its hospitality during a week's stay.
[1] A. Silvestri and M. Trodden, Rep. Prog. Phys. 72, 096901 (2009).

[2] T. Clifton, P. G. Ferreira, A. Padilla, and C. Skordis, Phys. Rep. 513, 1 (2012).

[3] E. J. Copeland, M. Sami, and S. Tsujikawa, Int. J. Mod. Phys. D 15, 1753 (2006).

[4] A. Lue, R. Scoccimarro, and G. D. Starkman, Phys. Rev. D 69, 124015 (2004).

[5] A. Joyce, B. Jain, J. Khoury, and M. Trodden, Phys. Rep. 568, 1 (2015).

[6] S. Tsujikawa, Lect. Notes Phys. 800, 99 (2010).

[7] E. Bertschinger and P. Zukin, Phys. Rev. D 78, 024015 (2008).

[8] L. Pogosian, A. Silvestri, K. Koyama, and G.-B. Zhao, Phys. Rev. D 81, 104023 (2010).

[9] I. Sawicki and E. Bellini, Phys. Rev. D 92, 084061 (2015).
[10] G. Gubitosi, F. Piazza, and F. Vernizzi, J. Cosmol. Astropart. Phys. 02 (2013) 032.

[11] J. K. Bloomfield, E. E. Flanagan, M. Park, and S. Watson, J. Cosmol. Astropart. Phys. 08 (2013) 010.

[12] J. Bloomfield, J. Cosmol. Astropart. Phys. 12 (2013) 044.

[13] J. Gleyzes, D. Langlois, F. Piazza, and F. Vernizzi, J. Cosmol. Astropart. Phys. 08 (2013) 025.

[14] J. Gleyzes, D. Langlois, and F. Vernizzi, Int. J. Mod. Phys. D 23, 1443010 (2014).

[15] N. Frusciante, M. Raveri, D. Vernieri, B. Hu, and A. Silvestri, Phys. Dark Universe 13, 7 (2016).

[16] N. Frusciante, G. Papadomanolakis, and A. Silvestri, J. Cosmol. Astropart. Phys. 07 (2016) 018.

[17] E. Bellini and I. Sawicki, J. Cosmol. Astropart. Phys. 07 (2014) 050. 
[18] J. Gleyzes, D. Langlois, F. Piazza, and F. Vernizzi, J. Cosmol. Astropart. Phys. 02 (2015) 018.

[19] G. W. Horndeski, Int. J. Theor. Phys. 10, 363 (1974).

[20] C. Deffayet, S. Deser, and G. Esposito-Farese, Phys. Rev. D 80, 064015 (2009).

[21] B. Abbott et al. (Virgo and LIGO Scientific Collaborations), Phys. Rev. Lett. 119, 161101 (2017).

[22] B. P. Abbott et al. (Virgo, Fermi-GBM, INTEGRAL, and LIGO Scientific Collaborations), Astrophys. J. 848, L13 (2017).

[23] D. A. Coulter et al., Science 358, 1556 (2017).

[24] P. Creminelli and F. Vernizzi, Phys. Rev. Lett. 119, 251302 (2017).

[25] J. M. Ezquiaga and M. Zumalacsrregui, Phys. Rev. Lett. 119, 251304 (2017).

[26] T. Baker, E. Bellini, P. G. Ferreira, M. Lagos, J. Noller, and I. Sawicki, Phys. Rev. Lett. 119, 251301 (2017).

[27] J. Sakstein and B. Jain, Phys. Rev. Lett. 119, 251303 (2017).

[28] D. Bettoni, J. M. Ezquiaga, K. Hinterbichler, and M. Zumalacrregui, Phys. Rev. D 95, 084029 (2017).

[29] R. Kase and S. Tsujikawa, arXiv:1809.08735.

[30] L. Heisenberg, arXiv:1807.01725.

[31] L. Amendola, D. Bettoni, G. Domnech, and A. R. Gomes, J. Cosmol. Astropart. Phys. 06 (2018) 029.

[32] L. Lombriser and A. Taylor, J. Cosmol. Astropart. Phys. 03 (2016) 031.

[33] L. Lombriser and N. A. Lima, Phys. Lett. B 765, 382 (2017).

[34] R. McManus, L. Lombriser, and J. Pearrubia, J. Cosmol. Astropart. Phys. 11 (2016) 006.

[35] E. J. Copeland, M. Kopp, A. Padilla, P. M. Saffin, and C. Skordis, Phys. Rev. Lett. 122, 061301 (2019).

[36] C. de Rham and S. Melville, Phys. Rev. Lett. 121, 221101 (2018).

[37] J. S. Y. Leung and Z. Huang, Int. J. Mod. Phys. D 26, 1750070 (2017).

[38] D. Alonso, E. Bellini, P. G. Ferreira, and M. Zumalacarregui, Phys. Rev. D 95, 063502 (2017).

[39] S. Casas, M. Kunz, M. Martinelli, and V. Pettorino, Phys. Dark Universe 18, 73 (2017).

[40] C. Heneka and L. Amendola, J. Cosmol. Astropart. Phys. 10 (2018) 004.

[41] A. S. Mancini, R. Reischke, V. Pettorino, B. M. Schaefer, and M. Zumalacarregui, Mon. Not. R. Astron. Soc. 480, 3725 (2018).

[42] C. Deffayet, O. Pujolas, I. Sawicki, and A. Vikman, J. Cosmol. Astropart. Phys. 10 (2010) 026.

[43] A. Silvestri, L. Pogosian, and R. V. Buniy, Phys. Rev. D 87, 104015 (2013).

[44] O. Pujolas, I. Sawicki, and A. Vikman, J. High Energy Phys. 11 (2011) 156.

[45] S. Peirone, K. Koyama, L. Pogosian, M. Raveri, and A. Silvestri, Phys. Rev. D 97, 043519 (2018).

[46] B. Hu, M. Raveri, N. Frusciante, and A. Silvestri, arXiv: 1405.3590.

[47] M. Chevallier and D. Polarski, Int. J. Mod. Phys. D 10, 213 (2001).

[48] E. V. Linder, Phys. Rev. Lett. 90, 091301 (2003).
[49] E. Bellini, A. J. Cuesta, R. Jimenez, and L. Verde, J. Cosmol. Astropart. Phys. 02 (2016) 053; 06 (2016) E01(E).

[50] A. De Felice and S. Tsujikawa, J. Cosmol. Astropart. Phys. 02 (2012) 007.

[51] L. Gergely and S. Tsujikawa, Phys. Rev. D 89, 064059 (2014).

[52] R. Kase and S. Tsujikawa, Phys. Rev. D 90, 044073 (2014).

[53] R. Kase and S. Tsujikawa, Int. J. Mod. Phys. D 23, 1443008 (2014).

[54] J. Gleyzes, D. Langlois, M. Mancarella, and F. Vernizzi, J. Cosmol. Astropart. Phys. 08 (2015) 054.

[55] A. De Felice, N. Frusciante, and G. Papadomanolakis, J. Cosmol. Astropart. Phys. 03 (2017) 027.

[56] N. Frusciante and G. Papadomanolakis, J. Cosmol. Astropart. Phys. 12 (2017) 014.

[57] N. Frusciante, G. Papadomanolakis, S. Peirone, and A. Silvestri, J. Cosmol. Astropart. Phys. 02 (2019) 029.

[58] C. D. Kreisch and E. Komatsu, J. Cosmol. Astropart. Phys. 12 (2018) 030.

[59] B. Hu, M. Raveri, N. Frusciante, and A. Silvestri, Phys. Rev. D 89, 103530 (2014).

[60] M. Raveri, B. Hu, N. Frusciante, and A. Silvestri, Phys. Rev. D 90, 043513 (2014).

[61] E. Bellini et al., Phys. Rev. D 97, 023520 (2018).

[62] N. Aghanim et al. (Planck Collaboration), Astron. Astrophys. 594, A11 (2016).

[63] P. A. R. Ade et al. (Planck Collaboration), Astron. Astrophys. 594, A13 (2016).

[64] P. A. R. Ade et al. (Planck Collaboration), Astron. Astrophys. 594, A15 (2016).

[65] S. Alam et al. (BOSS Collaboration), Mon. Not. R. Astron. Soc. 470, 2617 (2017).

[66] A. G. Riess et al., Astrophys. J. 826, 56 (2016).

[67] M. Betoule et al. (SDSS Collaboration), Astron. Astrophys. 568, A22 (2014).

[68] H. Hildebrandt et al., Mon. Not. R. Astron. Soc. 465, 1454 (2017).

[69] K. Kuijken et al., Mon. Not. R. Astron. Soc. 454, 3500 (2015).

[70] J. T. A. de Jong, G. A. V. Kleijn, K. H. Kuijken, and E. A. Valentijn, Exper. Astron. 35, 25 (2013).

[71] T. D. Kitching et al. (CFHTLenS Collaboration), Mon. Not. R. Astron. Soc. 442, 1326 (2014).

[72] P. A. R. Ade et al. (Planck Collaboration), Astron. Astrophys. 594, A14 (2016).

[73] M. Tegmark, A. Hamilton, M. Strauss, M. Vogeley, and A. Szalay, Astrophys. J. 499, 555 (1998).

[74] H.-J. Seo and D. J. Eisenstein, Astrophys. J. 665, 14 (2007).

[75] H.-J. Seo and D. J. Eisenstein, Astrophys. J. 633, 575 (2005).

[76] L. Amendola et al., Living Rev. Relativity 21, 2 (2018).

[77] R. Laureijs et al., arXiv:1110.3193.

[78] A. Aghamousa et al. (DESI Collaboration), arXiv: 1611.00036 .

[79] A. Aghamousa et al. (DESI Collaboration), arXiv: 1611.00037 . 
[80] R. Mandelbaum et al. (LSST Dark Energy Science Collaboration), arXiv:1809.01669.

[81] M. G. Santos, D. Alonso, P. Bull, M. Silva, and S. Yahya, arXiv:1501.03990.

[82] A. Raccanelli et al., Proc. Sci., AASKA14 (2015) 031.

[83] P. Bull, S. Camera, A. Raccanelli, C. Blake, P. G. Ferreira, M. G. Santos, and D. J. Schwarz, Proc. Sci., AASKA14 (2015) 024.

[84] P. Bull et al., arXiv:1810.02680.

[85] T. M. C. Abbott et al. (Dark Energy Survey Collaboration 1), Phys. Rev. D 98, 043526 (2018).

[86] R. Laureijs et al. (EUCLID Collaboration), arXiv: 1110.3193.

[87] Y. Wang, Mon. Not. R. Astron. Soc. 423, 3631 (2012).

[88] Y. Wang, C.-H. Chuang, and C. M. Hirata, Mon. Not. R. Astron. Soc. 430, 2446 (2013).

[89] V. Desjacques, D. Jeong, and F. Schmidt, Phys. Rep. 733, 1 (2018).

[90] D. J. Eisenstein, W. Hu, and M. Tegmark, Astrophys. J. 518, 2 (1999).

[91] N. Kaiser, Mon. Not. R. Astron. Soc. 227, 1 (1987).

[92] P. Bull, Astrophys. J. 817, 26 (2016).

[93] S. de la Torre and L. Guzzo, Mon. Not. R. Astron. Soc. 427, 327 (2012).

[94] S. Casas, M. Kunz, M. Martinelli, and V. Pettorino, Phys. Dark Universe 18, 73 (2017).

[95] L. Amendola, V. Pettorino, C. Quercellini, and A. Vollmer, Phys. Rev. D 85, 103008 (2012).
[96] S. Casas, M. Pauly, and J. Rubio, Phys. Rev. D 97, 043520 (2018).

[97] I. Harrison, S. Camera, J. Zuntz, and M. L. Brown, Mon. Not. R. Astron. Soc. 463, 3674 (2016).

[98] S. Hannestad and T. Schwetz, J. Cosmol. Astropart. Phys. 11 (2016) 035.

[99] F. Simpson, R. Jimenez, C. Pena-Garay, and L. Verde, J. Cosmol. Astropart. Phys. 06 (2017) 029.

[100] S. Vagnozzi, E. Giusarma, O. Mena, K. Freese, M. Gerbino, S. Ho, and M. Lattanzi, Phys. Rev. D 96, 123503 (2017).

[101] W. Yang, R. C. Nunes, S. Pan, and D. F. Mota, Phys. Rev. D 95, 103522 (2017).

[102] S. Peirone, N. Frusciante, B. Hu, M. Raveri, and A. Silvestri, Phys. Rev. D 97, 063518 (2018).

[103] E. V. Linder, Phys. Rev. D 95, 023518 (2017).

[104] F. Piazza, H. Steigerwald, and C. Marinoni, J. Cosmol. Astropart. Phys. 05 (2014) 043.

[105] L. Pogosian and A. Silvestri, Phys. Rev. D 94, 104014 (2016).

[106] J. Espejo, S. Peirone, M. Raveri, K. Koyama, L. Pogosian, and A. Silvestri, Phys. Rev. D 99, 023512 (2019).

[107] L. Perenon, F. Piazza, C. Marinoni, and L. Hui, J. Cosmol. Astropart. Phys. 11 (2015) 029.

[108] E. V. Linder, J. Cosmol. Astropart. Phys. 03 (2018) 005.

[109] S. Camera, in Proceedings of the 51st Rencontres de Moriond, Cosmology Session: La Thuile, Italy, 2016 (ARISF, La Thuile, Italy, 2016), pp. 331-336. 